\title{
Pessoas idosas e o Cuidado pós Covid-19
}

\author{
Older adults and Post-Covid-19 Care
}

Em dezembro de 2019 o mundo foi surpreendido pela descoberta de um novo vírus na cidade de Wuhan na China, que posteriormente foi denominado de SARS-CoV-2 (Severe Acute Respiratory Syndrome Coronavirus 2) e a doença em decorrência a este novo patógeno foi denominada de Covid-19 (Coronavirus Disease 2019). O surgimento da Covid-19 se mostrou como importante problema de saúde pública em todo o mundo diante do seu alto potencial de transmissibilidade e a sua importante letalidade, e desde então todas as nações estão em situação de alerta para o combate ao vírus e controle da doença ${ }^{1,2}$.

A propagação do vírus tomou proporções exponenciais e, em 11 de março de 2020, a Organização Mundial de Saúde (OMS) declarou a epidemia de Covid-19 como pandemia ${ }^{3}$.

As consequências da Covid-19 não se limitam apenas a infecções e mortes humanas, mas também têm repercussões sociais associadas, incluindo ataques raciais e xenófobos e implicações econômicas adversas ${ }^{4}$.

Apesar do vírus não escolher as especificidades pessoais, a Covid-19 tem se apresentado como uma doença que não possui predileção por idade, raça, condição socioeconômica ou nacionalidade, contudo, observouse uma alta incidência entre os adultos embora as maiores taxas de letalidade tenham sido registradas entre as pessoas com mais de 60 anos $^{5}$.

Os idosos apresentam-se como população de risco para as síndromes respiratórias agudas graves, e, portanto, precisam de um olhar atento sobre a sua condição de saúde ${ }^{6}$. A literatura aponta que são relatados diferentes graus de disfunções físicas, respiratórias e psicológicas em pacientes com Covid-19, especialmente entre os idosos ${ }^{7,8}$.

Tendo em vista que a Covid-19 é uma doença recente, o conhecimento a respeito do seu comportamento, principalmente no que se refere as suas sequelas e desfechos a longo prazo, ainda é bastante incipiente e tem inquietado diversos pesquisadores. É preciso considerar a possibilidade de surgimento de sequelas ou comprometimentos entre os pacientes que foram acometidos por esta doença, principalmente entre àqueles que a tiveram na forma mais grave e necessitaram de cuidados intensivos? ${ }^{9}$.

Em um estudo conduzido na Itália, antes do desenvolvimento da vacina, com participantes que se recuperaram da Covid-19 após um período de hospitalização, identificaram que independentemente da idade dos participantes, em $87,4 \%$ dos casos verificou-se a persistência de pelo menos um sintoma após o período de internação, sendo mais frequente a fadiga e a dispneia ${ }^{10}$. Nesta mesma perspectiva, os resultados de uma 
pesquisa desenvolvida em Israel evidenciaram que 79,8\% das pessoas que desenvolveram sequelas após a Covid-19 tinham mais de 60 anos $^{11}$. Os estudos reforçam que inúmeras pesquisas têm sido desenvolvidas com foco no período agudo da infecção, entretanto, é de extrema necessidade que seja realizado um monitoramento prolongado para que se reconheçam os efeitos a longo prazo.

Embora tenhamos avançado na vacinação da população idosa, inclusive com a aplicação da terceira dose, ainda não há total clareza acerca das consequências dessa doença na saúde das pessoas idosas.

Nesse contexto há a necessidade em (re)conhecer o comportamento da Covid-19 a longo prazo, em especial entre as pessoas idosas que se constituem como uma população de maior vulnerabilidade.

A pandemia de Covid-19, em especial no Brasil, tem colocado em evidência as iniquidades existentes e o quanto o país é discriminatório, sobretudo com as mulheres, os idosos, as pessoas com deficiência e com a população negra ${ }^{12}$. Portanto, é urgente que se produza conhecimento científico a respeito das consequências da Covid-19 para que as ações em saúde possam ser desenvolvidas de maneira efetiva, eficaz, resolutiva e que realmente atendam às necessidades de saúde das pessoas, sobretudo para a população idosa.

As experiências internacionais demonstram que as estratégias de atenção à Covid-19 centradas exclusivamente na atenção hospitalar não são suficientes para o cuidado integral às pessoas, reforçando, portanto, a necessidade de fortalecimento da Atenção Primária à Saúde (APS) visto que esta é a porta de entrada preferencial ao sistema de saúde e é o ponto de atenção que realizará o cuidado de maneira continua. Ao se considerar os atributos da APS vislumbra-se a potencialidade do cuidado ofertado neste nível de atenção para o acompanhamento e monitoramento da condição de saúde das pessoas que tiveram Covid-19 ${ }^{13}$.

Neste sentido, o reconhecimento do estado de saúde das pessoas idosas após a Covid-19 se torna de fundamental importância para que se conheça a real dimensão do impacto desta doença sobre a saúde das pessoas a longo prazo, e assim o cuidado possa ser planejado e ofertado de maneira integral e resolutiva.

Arthur de Almeida Medeiros'

\section{REFERÊNCIAS}

1. Wu D, Wu T, Liu Q, Yang Z. The SARS-CoV-2 outbreak: what we know. Int J Infect Dis [Internet]. 2020;89:9444-8. Disponível em: https://doi.org/10.1016/j.ijid.2020.03.004.

2. Rodriguez-Morales AJ, Cardona-Ospina JA, Gutiérrez-Ocampo E, Villamizar-Peña R, Holguin-Rivera Y, EscaleraAntezana JP, et al. Clinical, laboratory and imaging features of COVID-19: a systematic review and meta-analysis. Travel Med Infect Dis [Internet]. 2020;34:1-10. Disponível em: https://doi.org/10.1016/j.tmaid.2020.101623 . .

3. World Health Organization [Internet]. Geneva: WHO; 2020. Coronavírus disease 2019 (COVID-19). Situation Report - 51, [acesso em 18 mar 2020]. Disponível em: https://www.who.int/emergencies/diseases/novelcoronavirus-2019/situation-reports/ .

4. Shah S. A Commentary on "World Health Organization declares Global Emergency: a review of the 2019 Novel Coronavirus (COVID-19)”. Int J Surg [Internet]. 2020;76:128-9. Disponível em: https://doi.org/10.1016/j. ijsu.2020.03.001 .

Pós-Doutor em Saúde Coletiva, Docente do Mestrado Profissional em Saúde da Família do Instituto Integrado de Saúde da Universidade Federal de Mato Grosso do Sul. Campo Grande, MS, Brasil. 
5. Barbosa IR, Galvão MHR, Souza TA, Gomes SV, Medeiros AA, Lima KC. Incidence of and mortality from COVID-19 in the older Brazilian population and its relationship with contextual indicators: an ecological study. Rev Bras Geriatr Gerontol. 2020;23(1):e200171.

6. Garnier-Crussard A, Forestier E, Gilbert T, Krolak-Salmon P. Novel Coronavirus (COVID-19) Epidemic: What are the risks for older patients? J Am Geriatr Soc. 2020;68(5):1-2.

7. Liu K, Zhang W, Yang Y, Zhang J, Li Y, Chen Y. Respiratory rehabilitation in elderly patients with COVID-19: a randomized controlled study. Complement Ther Clin Pract. 2020;39:1-10.

8. Lai CC, Ko WC, Lee PI, Jean SS, Hsueh PR. Extra-respiratory manifestations of COVID-19. Int J Antimicrob Agents. 2020;56(2):1-10.

9. Sheehy LM. Considerations for Postacute Rehabilitation for Survivors of COVID-19. JMIR Public Health Surveill. 2020;6(2):e19462.

10. Carfi A, Bernabei R, Landi F. Persistent Symptoms in Patients After Acute COVID-19. JAMA. 2020;324(6):603-5.

11. Yanover C, Mizrahi B, Kalstein N, Marcus K, Akiva P, Barer Y, et al. What Factors Increase the Risk of Complications in SARS-CoV-2-Infected Patients? A Cohort Study in a Nationwide Israeli Health Organization. JMIR Public Health Surveill 2020;6(3):e20872.

12. Kalache A, Silva A, Giacomin KC, Lima KC, Ramos LR, Louvison M, et al. Aging and inequalities: social protection policies for older adults resulting from the Covid-19 pandemic in Brazil. Rev Bras Geriatr Gerontol 2020;23(6):e200122.

13. Medina MG, Giovanella L, Bousquat A, Mendonça, MHMD, Aquino R. Atenção primária à saúde em tempos de COVID-19: o que fazer? Cad Saúde Pública. 2020;36(6):e00149720. 